\title{
Fuzzy Logic Basis in High Performance Decision Support Systems
}

\author{
A. Bogdanov, A. Degtyarev, and Yu. Nechaev \\ Institute for High Performance Computing and Databases \\ Fontanka 118, 198005 St.-Petersburg, Russia \\ bogdanov@hm.csa.ru, deg@fn.csa.ru, int@fn.csa.ru
}

\begin{abstract}
The problem of synthesis of the onboard integrated intellectual complexes (IC) of decision support acceptance in fuzzy environment is discussed. The approach allowing to formalize complex knowledge system within the framework of fuzzy logic basis is formulated. Interpretation of fuzzy models is carried out with use of high performance computing at processing information stream in problems of analysis and forecast of worst-case situations (disputable and extreme).
\end{abstract}

\section{Introduction}

The present research is connected with use of soft computing concept in onboard integrated real time IC functioning. Formulation of practical recommendations and decisions acceptance in complex dynamic object (DO) control in extreme conditions is carried out on the basis of measurement data, mathematical models and the structured knowledge base (KB). The fundamental basis of research is theoretical principles of formalization of knowledge used in the organization of designing and systems functioning having in view their discrepancy and uncertainty, and also their algorithmic processing in decisions acceptance or in choice of operations for purposeful actions support. Practical realization of this problem is connected with development of effective algorithms of the analysis of the information obtained from measuring system and from procedural components of KB. Aspects of fuzzy logic allow to formalize discrepancy and uncertainty in the description of information and procedural elements of soft computing processes. Artificial neural networks (ANN) and genetic algorithms (GA) allow to organize training during calculations performance and their high-performance realization.

\section{Conceptual Model and IC Architecture}

The fundamental idea of employing IC for solving complex problems of DO interacting with the environment is connected with application of the measuring means and methods of analyzing the original information and supercomputer technology. 
The invariant kernel of the system includes the problem field, KB and DB of IC. For creation of this set conceptual model of IC is formed. The consideration of system in functional aspect allows to pick out the following components $[3,5]$ :

$$
<\mathrm{S}_{\mathrm{F}}, \mathrm{S}_{\mathrm{M}}, \mathrm{S}_{\mathrm{w}}>
$$

where $S_{F}=\left\{S_{F 1}, \ldots, S_{F N}\right\}$ is the set of functional subsystems; $S_{M}$ is structural scheme of the system; $S_{w}$ are conditions of formation of complete system;

$$
\mathrm{S}_{\mathrm{F}}=<\mathrm{X}, \mathrm{Y}, \mathrm{A}, \mathrm{P}_{\mathrm{F}}, \mathrm{T}_{\mathrm{F}}>, \mathrm{S}_{\mathrm{M}}=<\mathrm{E}, \mathrm{C}, \Phi, \mathrm{P}_{\mathrm{M}}, \mathrm{T}_{\mathrm{M}}>, \mathrm{S}_{\mathrm{W}}=<\mathrm{G}, \mathrm{R}, \mathrm{U}_{\mathrm{R}}, \mathrm{K}_{\mathrm{R}}, \mathrm{E}_{\mathrm{r}}>
$$

where $X=X_{j}(j=1, \ldots, n)$ is the vector-set of input signals; $Y=Y_{i}(i=1, \ldots, m)$ is the vector-set of output signals; $\mathrm{A}:\{\mathrm{X} \rightarrow \mathrm{Y}\}$ is the operator determining process of functioning of system $\mathrm{S} ; \mathrm{P}_{\mathrm{F}}=\left\{\mathrm{P}_{\mathrm{F}}^{\mathrm{x}}, \mathrm{P}_{\mathrm{F}}^{\mathrm{y}}, \mathrm{P}_{\mathrm{F}}^{\mathrm{A}}\right\}$ is the full set of functional parameters; $\mathrm{P}_{\mathrm{F}}^{\mathrm{x}}=\left\{\pi_{\mathrm{F}}^{\mathrm{x}}\right\} ; \mathrm{P}_{\mathrm{F}}^{\mathrm{y}}=\left\{\pi_{\mathrm{F}}^{\mathrm{y}}\right\} ; \mathrm{P}_{\mathrm{F}}^{\mathrm{A}}=\left\{\pi_{\mathrm{F}}^{\mathrm{A}}\right\}$ are the parameters for considered subsets; $\mathrm{T}_{\mathrm{F}}$ is the set of the time moments, invariant to object of modeling; $E=E_{v}\left(v=1, \ldots, N_{v}\right)$ is the set of system components; $\mathrm{C}=\mathrm{C}_{\mathrm{q}}\left(\mathrm{q}=1, \ldots, \mathrm{Q}_{\mathrm{c}}\right)$ is the set determining relations between elements; $\mathrm{P}_{\mathrm{M}}=\left\{\mathrm{P}_{\mathrm{M}}^{\mathrm{E}}, \mathrm{P}_{\mathrm{M}}^{\mathrm{C}}, \mathrm{P}_{\mathrm{M}}^{\Phi}\right\}$ is the set of morphological parameters. $\Phi=\left\{\Phi_{\lambda}\right\}(\lambda=1, \ldots, r)$ is the configuration set determining way of $S_{F}$ formation. $P_{M}^{E}=\left\{\pi_{M}^{E}\right.$ \}$; \mathrm{P}_{\mathrm{M}}^{\mathrm{C}}=\left\{\pi_{\mathrm{M}}^{\mathrm{C}}\right\} ; \mathrm{P}_{\mathrm{M}}^{\Phi}=\left\{\pi_{\mathrm{M}}^{\Phi}\right\}$ are the parameters for considered subsets; $\mathrm{T}_{\mathrm{M}}=\left\{\mathrm{t}_{\mathrm{k}}^{\mathrm{M}}\right\}$ is the set of the time moments of dynamic structures; $G$ are the purposes of functioning of object in realization of a task $R ; U_{R}$ are the principles and algorithms of management of object; $\mathrm{K}_{\mathrm{R}}$ is an execution result quality; $\mathrm{E}_{\mathrm{f}}$ is the efficiency determining at what price the purpose $\mathrm{G}$ is achieved.

Problem of selection of optimal requirements to quality operation index $\mathrm{Q}_{1}$ $(\mathrm{l}=1, \ldots, \mathrm{L})$ and of characteristics of IC tools $\mathrm{C}_{\mathrm{s}}(\mathrm{s}=1, \ldots, \mathrm{N})$ in conditions of incompleteness and indefiniteness of initial data is formulated on the basis on Zadeh' generalization principle [9]. In accordance with this principle possibilities of considered indexes realization are defined by the following:

for IC characteristics

$$
\mu_{S}\left(C_{S}\right)=\max _{C_{S 1}, \ldots, C_{S h}: C_{S}=f_{S}\left(C_{S 1} \ldots C_{S h}\right)} \mu_{S 1}\left(C_{S 1}\right) \Lambda \ldots \Lambda \mu_{S h}\left(C_{S h}\right), C_{S} \in R^{1}
$$

and for quality indexes

$$
\mu_{\Pi Q}\left(q_{l}\right)=\max _{C_{u 1}, \ldots, C_{u p}: q_{l}=Q_{l}\left(C_{u 1} \ldots C_{u p}\right)} \mu_{u 1}\left(C_{u 1}\right) \Lambda \ldots \Lambda \mu_{u p}\left(C_{u p}\right), q_{l} \in R^{1}
$$

with fuzzy sets

$$
\mathbf{C}_{\mathrm{s}}=\left\{\mathrm{C}_{\mathrm{s}}, \mu_{\mathrm{s}}\left(\mathrm{C}_{\mathrm{s}}\right)\right\} ; \quad \Pi \mathrm{Q}_{l}=\left\{\mathrm{q}_{l}, \mu_{\Pi q_{l}}\left(\mathrm{q}_{l}\right)\right\}
$$

It is possible to present optimal solution of problem for fuzzy goals $\mathrm{Q}$ and fuzzy possibilities PQ having in view conditions (2) and (3) as

$$
\mu_{\text {пеl }}\left(\mathrm{q}_{1}\right) \Lambda \mu_{\mathrm{Ql}}\left(\mathrm{q}_{1}\right) \rightarrow \max
$$


The realization of (1)-(4) is connected with the decision of the following functional tasks:

- diagnosing of the current condition of environment and dynamic object;

- recognition and classification of situation;

- the forecast of development of situation;

- search of control strategy;

- change of the current condition of dynamic object and forecast of its behavior under influence of the control decisions

Imitative modeling, procedure of the statistical and spectral analysis, filtration and forecast, identification and recognition of images are used as the mathematical tools for development of control algorithms.

The architecture of onboard IC is shown in the fig.1. The complex contains intelligence subsystems functioning on the basis of dynamic measurement data. Analysis and forecast of situations development and decisions acceptance are provided by means of the training system realizing adaptive component functions.

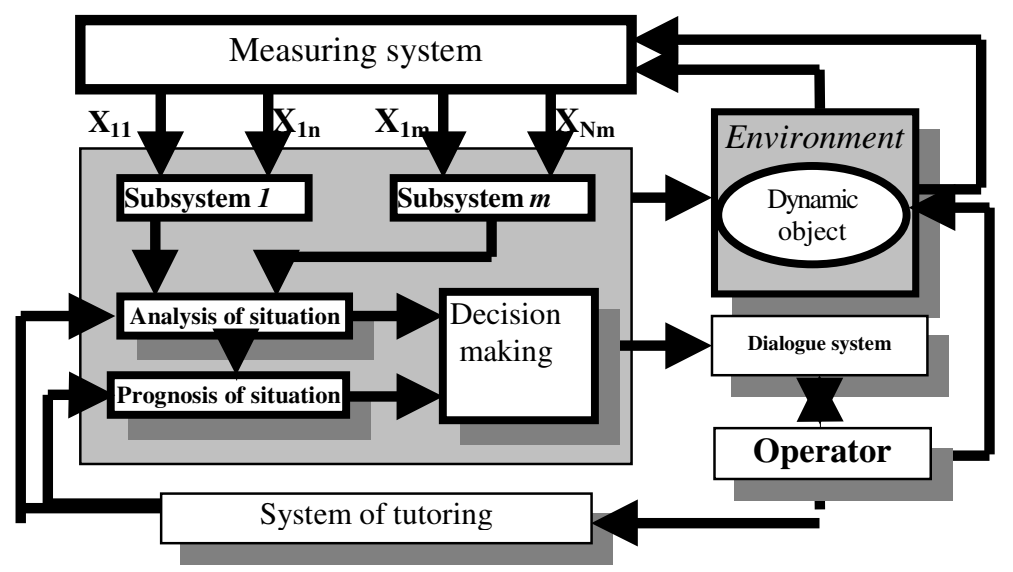

Fig. 1. Architecture of onboard intelligence complex

\section{Fuzzy Parallel Systems}

Development of IC software is based on the following artificial intellect paradigms. A basic element of information model of a considered subject domain is fuzzy production KB model. The conceptual model of IC functioning is formulated having in view requirements to information, computing and measuring technology. The formalized knowledge system is created by method of synthesis of control algorithm and decisions acceptance in conditions of uncertainty and incompleteness of the initial information.

Fuzzy logic inference is carried out with the help of modified modus ponens on the basis of the results of the current measurements [1], [8].

$$
\text { «If } X \text {, then } Y \text {, else } Z \text { » }(X \rightarrow Y(Z))
$$


where $X, Y, Z$ - are fuzzy sets defined on universal sets $U, V, W$. The sets $X, Y, Z$ are interpreted as a fuzzy input and output of some system. The relationship between these sets is defined by fuzzy system model.

The result of given conditional fuzzy operator completing (5) is characterized by the expression

$$
\Phi(\mathrm{X} \rightarrow \mathrm{Y}(\mathrm{Z}))=\left\{\mu_{\mathrm{x}} / \Phi(\mathrm{Y}),\left(1-\mu_{\mathrm{x}}\right) / \Phi(\mathrm{Z})\right\}
$$

where $\mu_{x}$ is truth degree of condition $X$.

Uniqueness of the defined group of operators is determined after calculating $\mu_{x}$ in accordance with given threshold of the truth degree $\gamma_{0} \in[0,1]$ :

$$
\Phi(\bullet)=\left(\Phi(Y) \text {, if } \mu_{\mathrm{x}} \geq \gamma_{0}\right) \text { or }\left(\Phi(Z) \text {, if } \mu_{\mathrm{x}}<\gamma_{0}\right)
$$

where $\Phi(\bullet)$ is a result of operators completing.

Specific feature of computing systems of new generation is orientation on knowledge processing and parallel functioning. Parallelism is inherent in knowledge processing systems. Therefore in perspective computer technologies design the problems of knowledge representation models adaptation to new computer architecture and hardware-software designing to suit highly effective parallel models of knowledge representation occur. Two approaches in fuzzy logic conclusion realization in onboard IC are used:

- Modus ponens based on minimax composition of entrance FS and the fuzzy relation "input-output" which describes a rule of decision acceptance;

- Situational inference based on fuzzy recognition of the situation by comparison of its description with the description of standard fuzzy situations.

Comparative analysis of these approaches is published in [12].

Development of methods and software of generation and alternatives choice on the basis of initial information on a situation being researched allows to rationally organize decision support system functioning. Analysis allows to determine set $\left(\mathrm{A}_{0}\right)$ of minimal dimension among known set of alternatives (A), each of which $\left(a_{n}\right)$ is estimated by one or several generalized criteria of quality $K_{q}$. Then, for any alternative $a_{i} \in \mathrm{A}$, the alternative $a_{j} \in \mathrm{A}_{0}$, concerning which conditions

$$
\mathrm{K}_{\mathrm{q}}\left(a_{\mathrm{j}} \in \mathrm{A}_{0}\right)>\mathrm{K}_{\mathrm{q}}\left(a_{\mathrm{i}} \in \mathrm{A}\right)
$$

are true is put in conformity.

The considered class of systems is characterized by the fact that for situations set there is a certain number of allowable decisions. Then the set of situations is broken into classes in such manner that the unique decision with necessary practical recommendations is put in conformity for each of these classes. The mechanism providing functioning logic and decisions development in process of entry of information on object dynamics and on environment is based on decisions conclusion with the help of formal procedure:

$$
F_{i}: S_{k}\left(t_{i}\right) \rightarrow U_{j}, k=1, \ldots, n ; j=1, \ldots, J ; i=1, \ldots, N, .
$$

based on the initial data concerning control decisions $\left\{u_{i}\right\}$. 


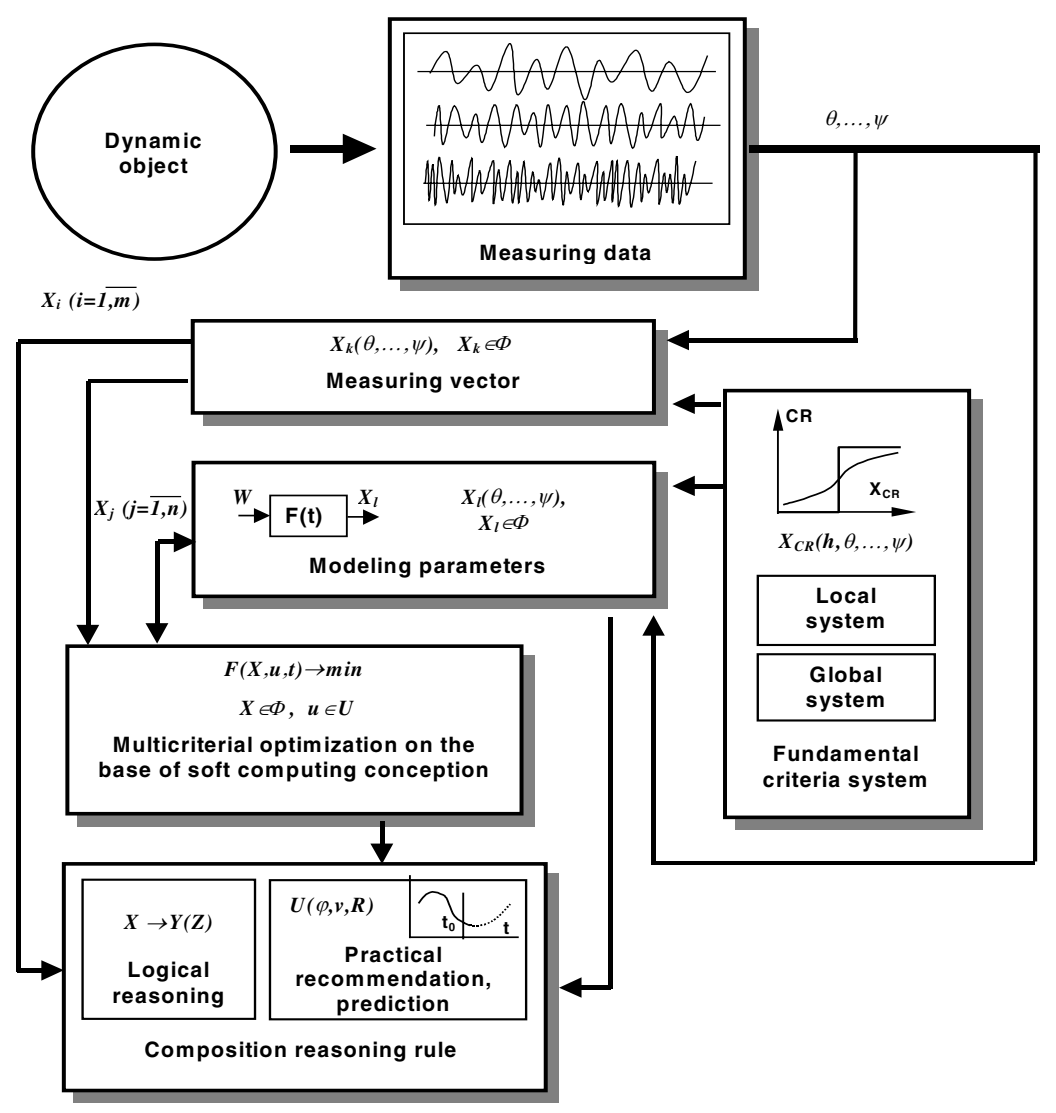

Fig. 2. Build-in data analysis and interpretation procedures in IC

In IC functioning in complex conditions inference rules usually contain the built in procedures of analysis and interpretation of measurement data (fig.2). In this case situations when the current measurement data result in various interpretation may arise. Then expansion of analogical inference rule [4] on fuzzy systems may be used. The approach developed by authors allows to carry out a fuzzy inference in the following sequence:

- the built-in procedures are realized and their efficiency is checked by mathematical modeling methods ;

- according to modeling results membership functions of antecedent parts are constructed;

- inference is carried out on the basis of model ( 5 )

As an example, let us consider fuzzy model of floating DO extreme situation identification by results of parameters $\theta, \psi, \zeta$ measurement (where $\theta$ is rolling, $\psi$ is pitching, $\zeta$ is heaving) in the case when antecedent part of rule is described by identical ratios 
$\mathrm{P}_{1}:<$ if $\Theta \in \mathrm{A}_{1} \& \psi \in \mathrm{A}_{2} \& \zeta \in \mathrm{A}_{3}$ then $\beta_{1} \in \mathrm{B}_{1}>$

$\mathrm{P}_{\mathrm{n}}:<$ if $\Theta \in \mathrm{A}_{1} \& \psi \in \mathrm{A}_{2} \& \zeta \in \mathrm{A}_{3}$ then $\beta_{\mathrm{n}} \in \mathrm{B}_{\mathrm{n}}>$;

$\mathrm{P}_{\mathrm{n}+1}:<$ if $\Theta^{\prime} \in \mathrm{A}_{1} \& \psi^{\prime} \in \mathrm{A}_{2} \& \zeta^{\prime} \in \mathrm{A}_{3}>$

$<\beta^{\prime} \in \mathrm{B}_{1}>$ - more likely.

Here $P_{1}, \ldots, P_{n}$ is group of situations allocated during search of identification problem decision; $P_{n+1}$ is the premise which is described on the basis of measurement results with the help of appropriate MF; $\left\langle\beta^{\prime} \in \mathrm{B}_{1}\right\rangle$ is the result which is determined during realization of fuzzy model (10) where MF of fuzzy set $\beta^{\prime}$ is calculated according to mathematical modeling for a situation characterized by higher truth degree (in this case for situation $\mathrm{P}_{1}$ ).

Using structure (5), it is possible to present result (10) as a composite rule

$\mathrm{Y}^{\prime}=\mathrm{X}^{\prime} \circ(\mathrm{X} \rightarrow \mathrm{Y})$;

$\mathrm{Y}^{\prime}=\mathrm{X}^{\prime} \circ \mathrm{R}$

$R=\left[r_{i j}\right](i=1, \ldots, m ; j=1, \ldots, n)$

and to realize parallel inference applying maximin composition and taking of minimum as fuzzy implication:

$$
\mu_{Y}=\bigcup_{x \in X} \mu_{X},(x) \Lambda \mu_{R}(x, y)=\bigcup_{x \in X} \mu_{X \cap X}(x) \Lambda \mu_{Y}(y)
$$

then MF is realized on basis of ANN.

One of complex problems in the organization of fuzzy inference procedure in parallel systems is preliminary processing of the measuring information and realization of procedures of data analysis and interpretation for fuzzy knowledge formation. This knowledge allows to construct MF of fuzzy hypothesis in inference models and also to provide realization of imitating modeling and statistical procedures of IC analysis in various operation conditions functioning especially in abnormal situations. Such calculations demand realization of high performance computing with the use of modern supercomputers [3]. Development of information processing algorithms in real time represents the important aspect at realization of IC designing computing technology requirements.

The paradigm of parallel calculations in realization of fuzzy logic inference assumes use of various models of approximate reasoning. As one of approaches it is possible to realize the algorithm offered in work [11]:

- preprocessor processing: construction of a matrix of parallelism and parallel sets $\Pi_{\mathrm{i}}(\mathrm{i}=1, \ldots, \mathrm{n})$ for all production models;

- initialization: input of initial condition $\mathrm{S}_{0}$ and installation of current value $\mathrm{S}=\mathrm{S}_{0}$; input of target condition $\mathrm{S}_{\mathrm{k}}$; if $\mathrm{S}=\mathrm{S}_{\mathrm{k}}$, then stop;

- search of the decision (the basic production cycle): calculation of activation set for current condition $\mathrm{S}$ (performance of a phase of production cycle comparison); determination of parallel carried out productions subset from $\cap \Pi_{i}$, and obtaining new current condition $S$; if $S \neq S_{k}$, then stop, otherwise return to the beginning of decision search stage. 
Interaction of rules in production system is fundamental concept in use of parallel processing. Parallel products activation is allowable at their mutual independence. Simultaneous performance of dependent rules may result in products interference $\mathrm{P}=\left\{\mathrm{P}_{\mathrm{q}}\right\}(\mathrm{q}=1, \ldots, \mathrm{Q})$ on to independent subsets.

A fuzzy neural network is one of the effective models for design of real time systems KB. Such model was offered by authors [2] for realization of algorithm of control vector formation of ship movement in ship-born IC. In this paper fuzzy production model is used as knowledge representation model. The fuzzy logic inference by results of current measurements is carried out with the help of the modified modus ponens

$$
\mu_{\sigma_{j}}=\max _{c} \min \left(\mu_{\sigma_{j}^{c}}, \min \operatorname{Poss}_{c, i}\right)
$$

where $\operatorname{Poss}_{c, i}\left(Z_{i}^{c}, \dot{Z}_{i}^{\prime}\right)=\sup _{Z} \min \left(\mu_{Z_{i}^{c}}, \mu_{Z_{i}^{\prime}}\right)$ is a measure of an opportunity.

Transformation of the entrance fuzzy variables (result of procedure (13)) in to an accurate estimation $\mathrm{u}$ is realized with the use of defuzzification procedure. The organization of antecedent parts of each rule calculations is carried out in fuzzy multilayered ANN. Basic elements of a network are fuzzy «AND» and «OR» formal neurones. During preprocessing an input vector calculation of measures of an opportunity (13) is stipulated. Values of synaptic weights are formed during training according to algorithm of optimization problem decision offered in work [13]. Elements of control vector are calculated on the basis of production model. Calculation by modus ponens is carried out in blocks of "MAX" operation. Accurate values of vector elements are formed according to defuzzification method in blocks $\ll d f »$.

Neural network approach to the calculations organization according to intellectual algorithm of ship control allows to provide IC functioning in real time. Increase of intellectuality degree of onboard integrated IC is achieved due to use of soft computing concept. Principles of fuzzy logic allow to formalize inaccuracy and uncertainty in the description of information and procedural elements of soft computing processes. ANN allow to organize training during calculations and high performance realization. The practical application of the soft computing concept at design of onboard IC for underwater device is considered in [10]. The KB of IC is represented as fuzzy models. Tabulated values of FM are stored in a database. The fuzzy inference is carried out with the help of modus ponens and procedure of accurate interpretation (defuzzification). Complexity of the intellectual control law has resulted in necessity of use of the computing procedures focused on performance in multiprocessor computing environment on the base of neural network models. Realization of this technology has allowed to set off optimum structures from the suboptimum decisions found during GA work. 


\section{Decisions Acceptance in Fuzzy Environment}

One of the most complex ways of on-board IC development is connected with development of tools ensuring functioning of integrated systems on the basis of a complex information flow. They include information from sensors of measuring system and results of mathematical modeling. The practical realization of the developed concept of analysis and forecast of dynamics of complex objects is carried out with using of supercomputer technologies ensuring the large resource of memory and speed. The functions of multiprocessor system are realized on the basis of the distributed architecture ensuring modularity of constructions and an opportunity of upgrading, reliability, distribution of loading and parallel processing of the information.

During IC functioning the control is carried out as a sequence of steps $u(\varphi, v, R)=u_{i}$ $(\mathrm{i}=1, \ldots, \mathrm{m})$. Local control goal is matched to each step.

As a result the dynamic object comes into certain condition $S(u)=S_{j}(j=1, \ldots, n)$ and has target signals $\mathrm{Y}(\mathrm{U}, \mathrm{R})=\mathrm{Y}_{\mathrm{k}}(\mathrm{k}=1, \ldots \mathrm{q})$. Quality of IC work to obtain practical recommendations on $\mathrm{r}^{\text {th }}$ a step is

$$
\Phi(r)=\Phi\left(S_{k}, S_{f}\right)
$$

where $\Phi(\mathrm{r})$ is error function; $S_{k}$ is technical condition of dynamic object (forecast); $S_{f}$ is actual technical condition

The value of error function is defined by reliability of the algorithms incorporated in formalized knowledge system.

To increase of efficiency of IC functioning the methods of the theory of experiment design are used. The problem of experiment design in real-time systems is connected with solution of the following problems:

- construction of optimum structure of mathematical models for adaptive components realization;

- choice of optimum conditions of measurements ensuring a reliable estimation of dynamic object characteristics and parameters of environment;

- development of algorithms of measuring information analysis

Algorithm of decision-making process defines a sequence of the procedures connected to interpretation of situations, development and analysis of alternatives, formulation of criteria, risk calculation and estimation, choice of way out variant , realization and check of the decision.

Object structurization as decisions tree allows to present logic decision functions of recognition, of the analysis and forecasting of considered situations. Construction of decisions tree subseting $\mathrm{A}_{\mathrm{k}}$ into $M$ images assumes:

- variables $\cdot$ set $X \in X_{k}(k=1, \ldots, N)$ with value areas $D_{1}, \ldots, D_{N}$ and $D_{1} \times \ldots \times D_{N}$

- variables set $\mathrm{x} \in \mathrm{X}_{\mathrm{k}}(a)$ for object $a \in \mathrm{A}_{\mathrm{k}}(\mathrm{k}=1, \ldots, \mathrm{N})$;

- separation of set $\mathrm{D}$ into pairs crossing subsets

$$
E^{1}, \ldots, E^{M}, E^{S}=E_{1}^{S_{1}} \times \ldots \times E_{k}^{S_{k}} S_{j} \in\left\{1, \ldots, \ell_{j}\right\}, S=1, \ldots, M, j=1, \ldots, k, k=1, \ldots, N
$$

In case of failure of initial information analysis with the help of traditional methods the decision is transferred to formation of the structure providing problem solution. The basis of such structure are the approximated methods and algorithms including 
approaches using nonconventional procedures (neural network logic basis, GA, etc.). In particular, algorithms of measuring system sensors parrying contain procedures of intelligence sensors creation basing on associative ANN and imitating modeling methods. Formation of the adaptive computing procedures which are taking into account volume and reliability of the initial data, makes one of the important trends in decision support systems design.

Process of decision support system synthesis is a sequence of hierarchical structure analysis operations. The elementary subtasks in this structure are analyzed preliminarily by method of hierarchies [7]. Realization of this method is connected to allocation of priorities (weights) of attributes with a view of choice of the best of them with the use of algebraic theory of matrixes and expert procedures:

$$
W \bar{\pi}=\lambda_{\max } \bar{\pi} ; \quad \Pi=\left[\begin{array}{ccc}
\pi_{11} & \cdots & \pi_{1 m} \\
\vdots & \pi_{i j} & \vdots \\
\pi_{n 1} & \cdots & \pi_{n m}
\end{array}\right] \times\left[\begin{array}{c}
g_{1} \\
\vdots \\
g_{m}
\end{array}\right],
$$

where $\mathrm{W}$ is the inverse symmetric matrix of values of paired comparisons of attributes concerning the given attribute; $\pi$ is the normalized vector of attributes weights; $\lambda_{\max }$ is the greatest eigenvalue of matrix $\mathrm{W}$; $\Pi$ is the result of determination of global priorities of attributes $\Pi_{1}, \ldots, \Pi_{\mathrm{N}} ; \mathrm{N}$ is the number of attributes; $\pi_{\mathrm{ij}}(\mathrm{i}=1, \ldots, \mathrm{n}$, $\mathrm{j}=1, \ldots, \mathrm{m})$ is the relative weight of $\mathrm{i}$-th attribute on $\mathrm{j}$-th attribute; $\mathrm{g}_{\mathrm{j}}$ is the relative weight of $j$-th attribute.

During such analysis attributes, to which the minimal global priority values correspond are allocated. The obtained data allow to generate space of the alternatives determining DO condition and control decisions, providing task performance. The result is achieved by finding compromise between the allocated space of alternatives $\mathrm{A}_{q}^{*}$ and attributes dictionary volume $\left\{\mathrm{x}_{\mathrm{i}}\right\}$. Realization of developed information technology in the analysis of extreme situations is shown in the fig. 3 .

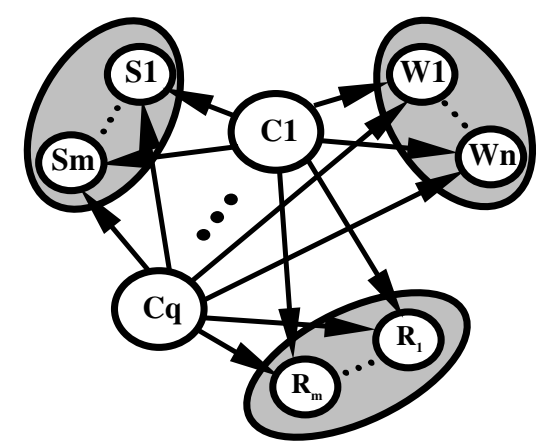

Fig.3 Fragment of a semantic network at the analysis of extreme situations

The semantic network presented here in which units frames are located, contains the following sets:

- $\mathrm{S}$ is the set of scripts containing the descriptions of dynamic stages and the appropriate updating in model parameters;

- $\mathrm{W}$ is the set of variants (DO conditions) described by information model; 
- $\mathrm{R}$ is the set of conclusions describing results of calculations of the script on given variant;

- $\mathrm{C}$ is the set of links uniting in a semantic network triads $<\mathrm{S}, \mathrm{W}, \mathrm{R}>$ and containing references to these sets

\section{Results of Experiments}

Practical realization of the developed technology is carried out as the integrated onboard complex of a navigation safety.

The IC full-scale tests have been carried out aboard a tanker in the Baltic Sea, aboard a container ship in a voyage in the Mediterranean and in the Atlantic and on a small ship in the Black Sea. The most complete investigation of the IC operation was checked on the small ship, where the ship motions on waves were chosen according to specially developed program.

In the course of the test operation the dynamic characteristics measurements, this system adaptation and completion of the designing process, receiving new knowledge about the ship behavior on seas and its integration with the old one were carried out. The following factors were of special practical interest: testing the reliability of the applied methods for estimating and predicting the seas parameters, the transverse stability characteristics and the $\mathrm{KB}$ reaction to the standard and non-standard situations arising in the seaway. All this allowed us to correct the mathematical models of the interaction of the ship and environment, the mechanism of logical output and the procedure of decision making on the basis of fuzzy aims and limitations.

The test proved the possibility of practical evaluation and prediction of the dynamic characteristics, of the reliability of $\mathrm{KB}$ operation under various conditions of service. The selective data of wave prediction are shown in [6], seaworthiness experiments are shown in table 1

Table 1. Comparison of seaworthiness characteristics

\begin{tabular}{|l|c|c|c|c|c|c|}
\hline Ship's type & \multicolumn{2}{|c|}{ Small ship } & \multicolumn{2}{c|}{ Container ship } & \multicolumn{2}{c|}{ Tanker } \\
\hline Speed, kn & 6 & 6 & 14.5 & 14.0 & 5 & 5 \\
\hline Course angle, deg & 45 & 90 & 10 & 10 & 0 & 0 \\
\hline Draught, m & 2.2 & 2.1 & 7.64 & 7.65 & 11.3 & 7.5 \\
\hline Wave length, m & 20 & 26 & 43.4 & 67.5 & 22 & 23 \\
\hline Wave height, m & 1.75 & 2.3 & 3.96 & 5.00 & 1.9 & 2.0 \\
\hline Rolling, deg & 12 & 16 & 5.6 & 6.5 & - & - \\
\hline Pitching, deg & 2.8 & 1.9 & 1.3 & 1.5 & - & - \\
\hline Actual GM, m & 0.84 & 0.83 & 0.530 & 0.525 & 0.8 & 1.8 \\
\hline Critical GM, m & 0.50 & 0.49 & 0.328 & 0.327 & 0.6 & 1.0 \\
\hline Heel on circ., deg & - & - & - & - & 3.2 & 9.6 \\
\hline Speed lost, kn & 3 & 1 & 2.3 & 2.8 & - & - \\
\hline
\end{tabular}




\section{Conclusion}

The real-time program complex on the basis of multiprocessor technologies presented in the paper is intended for the safety control of ships and offshore structures.

Developed IC solves complex and difficult for formalization problems of analysis and forecast of seaworthiness characteristics in dynamic area on a basis continuously entering in "on-line" measuring information. Methods used in IC have statistical and experimental basis. They are easily realized in onboard supercomputer and allow the further perfection on the basis of full-scale tests.

The work is supported by grant RFBR 00-07-90227.

\section{References}

1. Averkin A.N. at all. Fuzzy sets in control and artificial intelligence models. M.: Nauka, 1986 (in Russian)

2. Nechaev Yu., Degtyarev A., Siek Yu., Decisions making in real-time intelligence systems using soft computing concept.//Artificial Intelligence N3, 2000, pp.525-533 (in Russian, English version now is available in http://www.maus-soft.com/iai)

3. Nechaev Yu., Gorbachev Yu. Realization of complex intelligence systems based on supercomputer technology. //Proc. of International conference "Intelligence Multiprocessor Systems", IMS 99, Taganrog, 1999, pp.78-85 (in Russian)

4. Polya G., Mathematics and plausible reasoning. Princeton University Press, 1954.

5. Nechaev Yu., Degtyarev A. Account of peculiarities of ship's non-linear dynamics in seaworthiness estimation in real-time intelligence systems.//Proc. of the Int. conf. STAB 2000, Launceston, Tasmania, Australia, Feb. 2000, vol.2, pp.688-701

6. Nechaev Yu. The problem of wave parameters identification in ship's intelligence system.//Proc. of $13^{\text {th }}$ Int. conf. on hydrodynamics in ship design HYDRONAV'99, Sept. 1999, Gdansk, Poland, pp.201-208

7. Saaty T.L. A sealing method for priorities in hierarchical Structures //J.Math. Psychology, 1977. Vol.15. N3

8. Zadeh L.A. The concept of linguistic variable and its application to approximate reasoning. - N.Y.: Elsivieer P.C., 1973

9. Zadeh L Fuzzy logic, neural networks and soft computing//CACM,1994,v.37,N3,pp.77-84

10. Nechaev Yu.I., Siek Yu.L. Design of Ship-Board control system Based in the soft computing conception //Proc. of 11th Int. conf. on industrial and engineering applications of artificial intelligence and expert systems, IEA-98-AIE. Benicassim, Castellon, Spain, June,1998. Springer. Vol.11. pp.192--199.

11. Vagin V.N. Parallel inference in situation control systems //Proc. of the 1995 ISIC Workshop - $10^{\text {th }}$ IEEE International Symposium on Intelligent Control. Aug.1995 Monterey (Cal.) USA, pp.109-116

12. Melikhov A.N., et al. Situation advised systems with fuzzy logic. M., Nauka, 1990 (in Russian)

13. Pedrycz W. Fuzzy neural networks with reference neurons as classifiers. // IEEE Trans. Neural Networks, vol. 3, 1992, pp. 770-775. 